\title{
Artifact-free Imaging of Photolithographic Masks by Environmental Scanning Electron Microscopy
}

\author{
M. Toth,* B.L. Thiel, ** M.A. Coy, ${ }^{*}$ J.G. Marshman* and W.R. Knowles* \\ * FEI Company, 1 Corporation Way \#2, Peabody, MA, USA 01960. \\ ** College of Nanoscale Science and Engineering, University at Albany - SUNY, 255 Fuller Rd, \\ Albany, NY, USA 12203
}

High resolution secondary electron imaging of photomasks is demonstrated using immersion magnetic lens, environmental scanning electron microscopy (ESEM). Charging artifacts and the buildup rate of carbonaceous contaminants (produced by electron induced polymerization or crosslinking of mobile hydrocarbons at the sample surface) are minimized though optimal detector design and appropriate selection of imaging gas.

Imaging of photolithographic masks by scanning electron microscopy is often complicated by chronic charging [2,3]. Masks consist of an insulating substrate that is partially covered by an electrically conductive thin film. In general, the substrate spans an area of $231 \mathrm{~cm}^{2}$, and is $6.25 \mathrm{~mm}$ tall. Absorber materials include chromium $(\mathrm{Cr})$, molybdenum silicide $(\mathrm{MoSi})$ and tantalum nitride $(\mathrm{TaN})$. The absorber is approximately $75-100 \mathrm{~nm}$ tall, and lateral feature dimensions extend down to the sub-400 nm range. Typically, the vast majority of the absorber features are not connected to each other and can not be grounded.

During electron imaging, the severity of charging artifacts depends strongly on the uniformity of the electric potential field, $\mathrm{V}_{\mathrm{S}}(\mathrm{x}, \mathrm{y})$ across the sample surface. This, in turn, depends on the geometry of the absorber within and in the neighborhood of the imaged region. Consequently, regions of masks containing large, symmetrical features (e.g., absorber islands running across the entire field of view) give rise to charging that is relatively easy to control using techniques such as the total yield method [4] employed in low voltage SEM. Conversely, isolated, small absorber islands and asymmetric absorber geometries can give rise to a highly non-uniform surface potential, and severe image distortions that can not be eliminated using high vacuum SEM. In such instances, the fluctuations in $\mathrm{V}_{\mathrm{S}}(\mathrm{x}, \mathrm{y})$ can be reduced using ESEM, where charging is controlled by ionized gas molecules. The ions serve to reduce variations in $\mathrm{V}_{\mathrm{S}}(\mathrm{x}, \mathrm{y})$ because they are low energy free carriers whose concentration at and above the sample surface is affected strongly by $\mathrm{V}_{\mathrm{S}}(\mathrm{x}, \mathrm{y})$ [5]. However, the efficiency of ion-induced charge control is limited by the detector geometry for the following reasons:

- The ions irradiate a macroscopic region of the sample, making $\mathrm{V}_{\mathrm{S}}(\mathrm{x}, \mathrm{y})$ dependent on the geometry of the absorber within a large area around the imaged region.

- $\mathrm{V}_{\mathrm{S}}(\mathrm{x}, \mathrm{y})$ is time-dependent due to charging up and discharging of mask regions within the field of view, as the beam is scanned during imaging.

- The ion concentration at and above the sample surface is a function of the detector electric field. Consequently, while $\mathrm{V}_{\mathrm{S}}$ may be uniform across the sample surface, it can float to voltages on the order of $10^{2} \mathrm{~V}[6,7]$.

Artifacts produced by the above effects can be minimized using electrode structures designed to collect excess ions [6]. This technique entails directing the ions to a sink (i.e., a cathode) through a path on which the ion trajectories are susceptible to subtle variations in the electric field produced by 
specimen charging and by excess ions at the sample surface. Implementation of such methods on ultra-high resolution magnetic immersion lens columns [7] permits attainment of secondary electron images such as the one shown in Fig. 1, obtained from a region of mask that charged severely in high vacuum. The image contains an opaque bridge defect site that had been etched using an electron beam repair process. The images shown in Fig. 1 and 2 illustrate the absence of charging artifacts on mask regions containing a range of absorber geometries.

ESEM is known to exhibit low contamination buildup rates [2]. This is illustrated by the images shown in Fig. 2, where a single region of sample was imaged consecutively at two magnifications (image acquisition time $=11.2 \mathrm{~s}$ ). The reduced magnification image does not contain a contamination square corresponding to the area imaged in (a).

Efficient hydrocarbon contamination reduction requires correct selection of ESEM imaging gas. This has been illustrated by analyses of electron beam-induced, metallic deposits produced in gaseous environments, and in high vacuum [8]. Unambiguous identification of the mechanisms behind the contamination reduction effect requires further work. Appropriate selection of gas type is also required for high quality secondary electron imaging and stable charge control. Specifically, the gas must behave as an efficient, stable gas cascade amplifier, and must not scatter the beam excessively. Water vapor is an example of a gas satisfying all of the above requirements. The benefits and shortcomings of other gases will be discussed.

\section{References}

[1] J.J. Hern, In: J.J. Hern et al (eds.), Introduction to Analytical Electron Microscopy, Plenum Press, New York (1979) 481.

[2] M.T. Postek et al., J. Microlith., Microfab., Microsyst. (JM $\left.{ }^{3}\right) 3$ (2004) 224.

[3] D.C. Joy, Proc. SPIE Int. Soc. Opt. Eng. 5375 (2004) 10.

[4] D.C. Joy \& C.S. Joy, Micron 27 (1996) 247.

[5] M. Toth et al., J. Appl. Phys. 91 (2002) 4479.

[6] J.P. Craven et al., J. Microsc. 205 (2002) 96.

[7] W.R. Knowles et al. (2005) elsewhere in these proceedings.

[8] A. Folch et al., J. Vac. Sci. Technol. B 14 (1996) 2609.

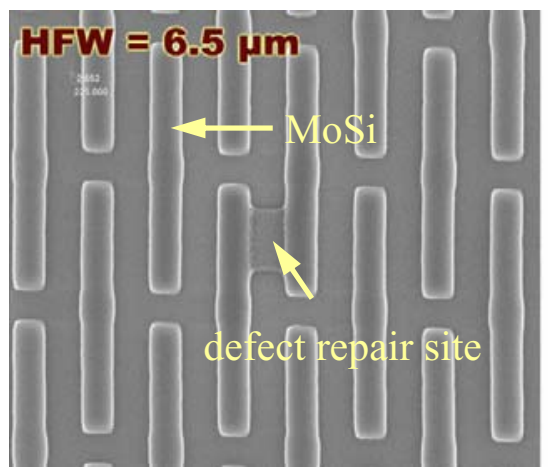

FIG. 1. ESEM image of a MoSi on quartz photomask, containing an opaque bridge defect repair site
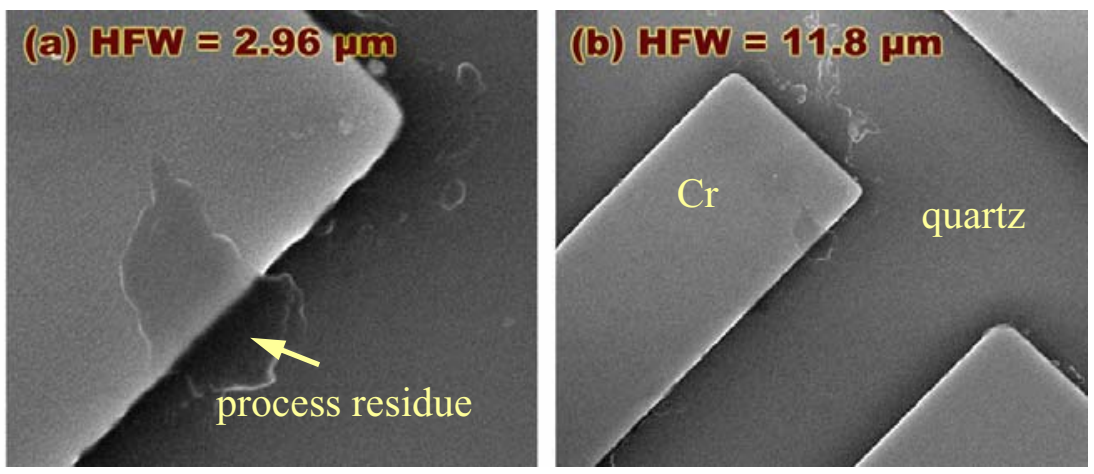

FIG. 2. ESEM images of a $\mathrm{Cr}$ on quartz photomask, acquired at two magnifications [HFW $=$ horizontal field width.] 